\title{
BMJ Open Randomised controlled trial of topical kanuka honey for the treatment of rosacea
}

Irene Braithwaite, ${ }^{1}$ Anna Hunt, ${ }^{1}$ Judith Riley, ${ }^{1}$ James Fingleton, ${ }^{1}$ Janwillem Kocks, ${ }^{1}$ Andrew Corin, ${ }^{2}$ Colin Helm, ${ }^{2}$ Davitt Sheahan, ${ }^{3}$ Christopher Tofield, ${ }^{4}$ Barney Montgomery, ${ }^{5}$ Mark Holliday, ${ }^{1}$ Mark Weatherall, ${ }^{6}$ Richard Beasley ${ }^{1}$

To cite: Braithwaite I, Hunt A, Riley J, et al. Randomised controlled trial of topical kanuka honey for the treatment of rosacea. BMJ Open 2015;5: e007651. doi:10.1136/ bmjopen-2015-007651

- Prepublication history and additional material is available. To view please visit the journal (http://dx.doi.org/ 10.1136/bmjopen-2015007651).

Received 12 January 2015 Revised 4 May 2015 Accepted 7 May 2015

\section{(a) CrossMark}

${ }^{1}$ Medical Research Institute of New Zealand, Wellington, New Zealand

${ }^{2}$ Clinical Horizons, Tauranga, New Zealand

${ }^{3}$ Papamoa Pines Medical

Centre, Tauranga, New

Zealand

${ }^{4}$ Cameron Medical Clinic

Tauranga, New Zealand

${ }^{5}$ Optimal Clinical Trials,

Auckland, New Zealand

${ }^{6}$ University of Otago,

Wellington, New Zealand

Correspondence to Dr Irene Braithwaite; Irene.braithwaite@mrinz.ac.nz

\section{ABSTRACT}

Objective: To investigate the efficacy of topical $90 \%$ medical-grade kanuka honey and $10 \%$ glycerine (Honevo) as a treatment for rosacea.

Design: Randomised controlled trial with blinded assessment of primary outcome variable.

Setting: Outpatient primary healthcare population from 5 New Zealand sites.

Participants: 138 adults aged $\geq 16$, with a diagnosis of rosacea, and a baseline blinded Investigator Global Assessment of Rosacea Severity Score (IGA-RSS) of $\geq 2$. 69 participants were randomised to each treatment arm. 1 participant was excluded from the Honevo group, and 7 and 15 participants withdrew from the Honevo and control groups, respectively.

Interventions: Participants were randomly allocated 1:1 to Honevo or control cream (Cetomacrogol), applied twice daily for 8 weeks.

Main outcome measures: The primary outcome measure was the proportion of participants who had a $\geq 2$ improvement in the 7-point IGA-RSS at week 8 compared to baseline. Secondary outcomes included change in IGA-RSS and subject-rated visual analogue score of change in severity (VAS-CS) on a $100 \mathrm{~mm}$ scale (0 mm 'much worse', 100 mm 'much improved') at weeks 2 and 8.

Results: $24 / 68$ (34.3\%) in the Honevo group and 12/ $69(17.4 \%)$ in the control group had $\mathrm{a} \geq 2$ improvement in IGA-RSS at week 8 compared to baseline (relative risk 2.03; $95 \% \mathrm{Cl} 1.11$ to $3.72, p=0.020$ ). The change in IGA-RSS for Honevo compared to control at week 2 minus baseline was -1 (Hodges-Lehman estimate, 95\% $\mathrm{Cl}-1$ to $0, p=0.03$ ), and at week 8 minus baseline was -1 (Hodges-Lehman estimate, $95 \% \mathrm{Cl}-1$ to 0 , $\mathrm{p}=0.005)$. The VAS-CS at week 2 was $9.1(95 \% \mathrm{Cl} 3.5$ to 14.7$), p=0.002$, and at week 8 was $12.3(95 \% \mathrm{Cl} 5.7$ to 18.9$), p<0.001$ for Honevo compared to control. Conclusions: Honevo is an effective treatment for rosacea.

Trial registration number: This trial was registered in the Australian and New Zealand Clinical Trials Registry ACTRN12614000004662.

\section{INTRODUCTION}

Rosacea is a common chronic inflammatory skin condition which primarily affects the
Strengths and limitations of this study

- This is a randomised placebo-controlled trial of 8 weeks of treatment for rosacea with either topical $90 \%$ medical-grade kanuka honey and $10 \%$ glycerine cream (Honevo) or a non-ionic paraffin-based cream (cetomacragol).

- Owing to the nature of the products being assessed, participants were not able to be blinded to the treatment arms.

- The primary outcome variable for this study (Investigator Global Assessment of Rosacea Severity Score) was assessed by investigators who were blinded to randomisation throughout the trial.

face, and occurs in up to $10 \%$ of the adult population. ${ }^{1-4}$ There is no cure, and affected individuals may experience substantial morbidity. There is a range of treatment options, including several topical and oral antibiotics, however, these are only partially effective and side effects may limit their use ${ }^{4-7}$ Also, there are global concerns about the increasing rates of resistance to antibiotics resulting from their widespread use, particularly with long-term use in chronic conditions. ${ }^{8}{ }^{9}$ For example, the United Kingdom Standing Medical Advisory Committee now recommends the fewest number of antibiotic courses should be prescribed for the shortest period possible. $^{10}$

Among the alternative therapies to antibiotics, medical-grade kanuka honey is of interest due to its potent antibacterial and anti-inflammatory activities. ${ }^{11-15}$ The pathophysiological rationale underlying its use is that rosacea is an inflammatory disorder, and that antigenic proteins related to the bacterium Bacillus oleronius isolated from the Demodex folliculorum mite, which infests the skin in rosacea, exacerbates this inflammatory response. ${ }^{16}{ }^{17}$ Furthermore, people with rosacea express abnormally high levels of the antimicrobial peptide cathelicidin, which promotes the inflammatory response in rosacea. $^{18}$ 
A recent pilot study of topical medical-grade kanuka honey as a treatment for rosacea found it to be an acceptable and potentially effective treatment. ${ }^{19}$ The addition of $10 \%$ glycerine to the honey has resulted in a product that is easier to apply to the skin. In this randomised controlled trial, we have investigated the efficacy of kanuka honey in the treatment of rosacea. We designed the trial to overcome the recognised limitations of previous studies, in particular to ensure that there was blinded investigator assessment of rosacea severity. ${ }^{6}$

\section{METHODS}

This parallel group, randomised, controlled trial with assessor blinding was undertaken at a hospital-based research facility and four community-based research and/ or primary care sites in New Zealand. Ethics approval was obtained from the Central Health and Disability Ethics Committee (13/CEN/118). Adults aged 16 or over with a doctor's diagnosis of rosacea on the face, and a baseline blinded Investigator Global Assessment of Rosacea Severity Score (IGA-RSS) of facial rosacea of $\geq 2$ were recruited. The IGA-RSS is a 7-point scale (from 0: 'clear', to 6: 'severe') that provides an integrated assessment of rosacea severity based on the principal facial signs of papules/pustules, inflammatory lesions, erythema and telangiectasia ${ }^{5}$ (see online supplementary table S1). Participants were identified at the time of first presentation or, with their primary care practitioner's consent, from pre-existing databases, or by public advertisement.

Exclusion criteria included current requirement for systemic corticosteroids, or systemic corticosteroid treatment in the 4 weeks prior to visit 1 , current requirement for oral or topical antibiotic therapy for rosacea, current requirement for topical corticosteroid treatment for rosacea, known or suspected allergy to honey, or Cetomacrogol control cream, or any other condition which, at the investigators discretion, it was believed may present a safety risk or impact the feasibility of the study or the study results.

Participants attended for 3 visits (see online supplementary table S2). Visit 1 (week 0) consisted of consent, baseline assessments (the IGA-RSS), a participant-rated rosacea severity visual analogue score (VAS-S) on a $100 \mathrm{~mm}$ scale $(0 \mathrm{~mm}$ being 'mildest possible' symptoms and $100 \mathrm{~mm}$ being 'worst possible' symptoms), and a participant-rated dermatology quality of life index (DLQI), ${ }^{20}$ followed by randomisation to Honevo or Cetomacrogol cream (control). At visit 2 (week 2) and visit 3 (week 8), as well as the IGA-RSS and the DLQI, participants completed a subjective rosacea 'change in severity' visual analogue scale (VAS-CS) on a $100 \mathrm{~mm}$ scale ( $0 \mathrm{~mm}$ being 'much worse' and $100 \mathrm{~mm}$ being 'much improved'). Participants' diaries were used throughout the study to capture each participant's weekly subjective VAS-S, their use of randomised treatment throughout the 8-week study period, and any general comments including adverse events throughout the study.

\section{Randomisation and blinding}

Treatment allocation was randomised using a computer generated sequence concealed to investigators by enclosing the proposed treatment arm in an opaque envelope that was opened only by primary investigators after informed consent was obtained from each participant. Participants were randomised in a 1:1 ratio to the topical application of Honevo or control cream. Owing to the nature of Honevo, it was not possible to blind the participants and primary investigators to the treatment allocation. An independent investigator at each site remained blinded to the treatment allocation throughout the study to perform the blinded IGA. The blinded investigator undertook only the IGA-RSS assessment for this study, and was not involved in any other study procedures. Participants were instructed not to communicate with the blinded investigator during the assessments.

\section{Randomised treatments}

The Investigational Product was topical medical-grade kanuka honey with $10 \%$ glycerine content (Honevo). The control cream was Cetomacrogol, a liquid paraffin and white soft paraffin topical emollient. ${ }^{21} 22$ The participants were instructed to apply an appropriate amount of cream to the affected area twice daily for 30-60 $\mathrm{min}$ per application, for 8 weeks, and to remove the treatment with warm water as desired. Participants were asked not to use any additional treatment for their rosacea for the duration of the study, as per the exclusion criteria.

\section{Outcome measures}

The primary outcome measure was the proportion of participants who had a $\geq 2$ improvement (reduction) in the IGA-RSS at week 8 (designated 'responders'). This measure represents a clinically meaningful improvement in severity of rosacea. The secondary outcome measures included the change from baseline in IGA-RSS at weeks 2 and 8; the participant-rated VAS-CS at weeks 2 and 8; the change from baseline in participant-rated VAS-S at weeks 2 and 8; the weekly diary-documented Rosacea severity VAS-S from participant diaries; the change from baseline in the participant-rated $\mathrm{DLQI}^{20}$ at weeks 2 and 8; withdrawals due to worsening of Rosacea; adverse events; and the daily self-reported use of Honevo (applications per day). Data for all participants was included for analysis up until the time the participant withdrew from the study or became ineligible due to the use of prohibited medications.

\section{Sample size and study power}

We anticipated the proportion of participants in the control group who respond with a $\geq 2$ reduction in blinded IGA would be between $25 \%$ and $50 \%{ }^{6}$ A total of 124 participants (62 in each group) has $80 \%$ power at $5 \%$ significance to detect a $25 \%$ response rate in the control group, and a 50\% response rate in the Honevo 
group. We recruited 138 participants to allow for a $10 \%$ drop-out rate.

\section{Statistical methods}

The study was analysed by an intention to treat, with the participants who withdrew considered to be nonresponders. The prespecified statistical analysis was logistic regression for the difference in proportions with response.

Relative risks for a $\geq 2$ point change in IGA-RSS at week 8 from baseline and for total study withdrawal were calculated, with $\mathrm{p}$ values using Fisher's exact test. ORs were also calculated from logistic regression

For the Likert-scaled variables, the Wilcoxon test, and Hodges-Lehman estimator of location shift for the difference between treatments were used. DLQI and VAS variables were analysed by analysis of covariance (ANCOVA) with the baseline value as a continuous covariate and the randomisation as the main predictor variable. The estimates for these analyses are interpreted as the difference between randomised groups adjusted for baseline.

Applications per day were analysed by analysis of variance (ANOVA) with the response variable as the mean average number of applications per day, predictor variable randomisation group, and using the number of days in the trial as a weight, to account for variations in the number of days of application.

In a post hoc analysis, the proportion of participants in whom the IGA-RSS was zero (clear of rosacea) at week 8 was calculated.

SAS V.9.3 was used.

\section{RESULTS}

The flow of participants in the study is shown in figure 1. There were 69 participants randomised to control and 69 to Honevo. One Honevo participant was subsequently excluded due to the use of a prohibited medication on enrolment, and their data was not used in the consequent analysis. The characteristics of the participants are shown in table 1 . Participants were predominantly aged between 50 and 70 years, and had had rosacea for a mean of 15 years. Nineteen per cent of participants in each group had previously used oral antibiotics for rosacea, while $44 \%$ and $38 \%$ had previously used any topical treatments for rosacea in the Honevo and control groups, respectively. There were 7/68 (10.3\%) withdrawals in the Honevo group (3 worsening rosacea, 2 took prohibited medications, 2 for other reasons unrelated to the study) and 15/69 (21.7\%) withdrawals in the control group (8 worsening rosacea, 2 took prohibited medications, 1 did not want to take the control medication, 1 found the study inconvenient, and 3 for reasons unrelated to the study).

\section{Primary outcome}

There were 24/68 (34.3\%) in the Honevo group and $12 / 69(17.4 \%)$ in the control group who had a $\geq 2$ improvement in IGA-RSS at week 8 compared to baseline (relative risk 2.03 (95\% CI 1.11 to 3.72), $\mathrm{p}=0.020$. The corresponding OR was 2.59 (1.17 to 5.74).

\section{Secondary outcomes}

The change from baseline in IGA-RSS for participants who did not withdraw is shown in table 2, online supplement table S3 and figure 2. The change in IGA-RSS for Honevo compared to control at week 2 minus baseline was -1 (Hodges-Lehman estimate, 95\% CI -1 to 0 , $\mathrm{p}=0.03$ ), and at week 8 minus baseline was -1 (Hodges-Lehman estimate, 95\% CI -1 to $0, \mathrm{p}=0.005$; table 3). The subject-rated VAS-CS at week 2 was 9.1 (CI 3.5 to 14.7 ), $\mathrm{p}=0.002$, and at week 8 was 12.3 (CI 5.7 to 18.9), $\mathrm{p}<0.001$ for Honevo compared to control, representing greater improvement with Honevo. There was no significant difference in diary-captured VAS-S, adjusted for baseline, at any of the time points between weeks 2 and 8 (see online supplementary table S4). There was no significant difference in the participant-rated DLQI adjusted for baseline at week 2 ( -0.3 , CI -1.1 to 0.6 , $\mathrm{p}=0.51)$ or week $8(-0.01, \mathrm{CI}-0.7$ to $0.7, \mathrm{p}=0.97)$.

The number of applications per day between the randomised treatments was similar (mean (SD) $1.84(0.23)$ vs $1.86(0.20)$ for the Honevo and control groups, respectively, difference: -0.02 (95\% CI -0.10 to 0.05 ), $\mathrm{p}=0.55)$.

In a post hoc analysis, the proportion of participants in whom the IGA-RSS score at week 8 was zero, (ie, full resolution of rosacea) was 9/68 (13.2\%) and 2/69 $(2.9 \%)$ in the Honevo and control groups, respectively, relative risk 4.6 (95\% CI 1.0 to $20.4, \mathrm{p}=0.031)$.

In the Honevo group, 23 participants reported 31 adverse events; 17 rosacea related, (3 of which resulted in withdrawal of the participants), and 14 unrelated to rosacea (see online supplementary table S5). In the control group, 27 participants reported 36 adverse events; 22 rosacea related, ( 8 of which resulted in withdrawal of participants), and 14 unrelated to rosacea.

\section{DISCUSSION}

This randomised controlled trial has demonstrated that topical $90 \%$ medical-grade kanuka honey and $10 \%$ glycerine (Honevo) is an effective and well tolerated treatment for rosacea. About one-third of participants had a clinically significant improvement in the IGA-RSS after 8 weeks of Honevo treatment, twofold greater than that observed with the control treatment. We recommend consideration of the use of kanuka honey as a treatment for rosacea.

There are a number of methodological issues that are important in the consideration of the study findings. There are no standard validated tools for assessing the severity of rosacea, which is inherently difficult due to its varied clinical characteristics. The priority with this study of a honey product was to reduce potential bias by blinding of clinical assessments where possible, as the 
Figure 1 Flow of participants through trial.

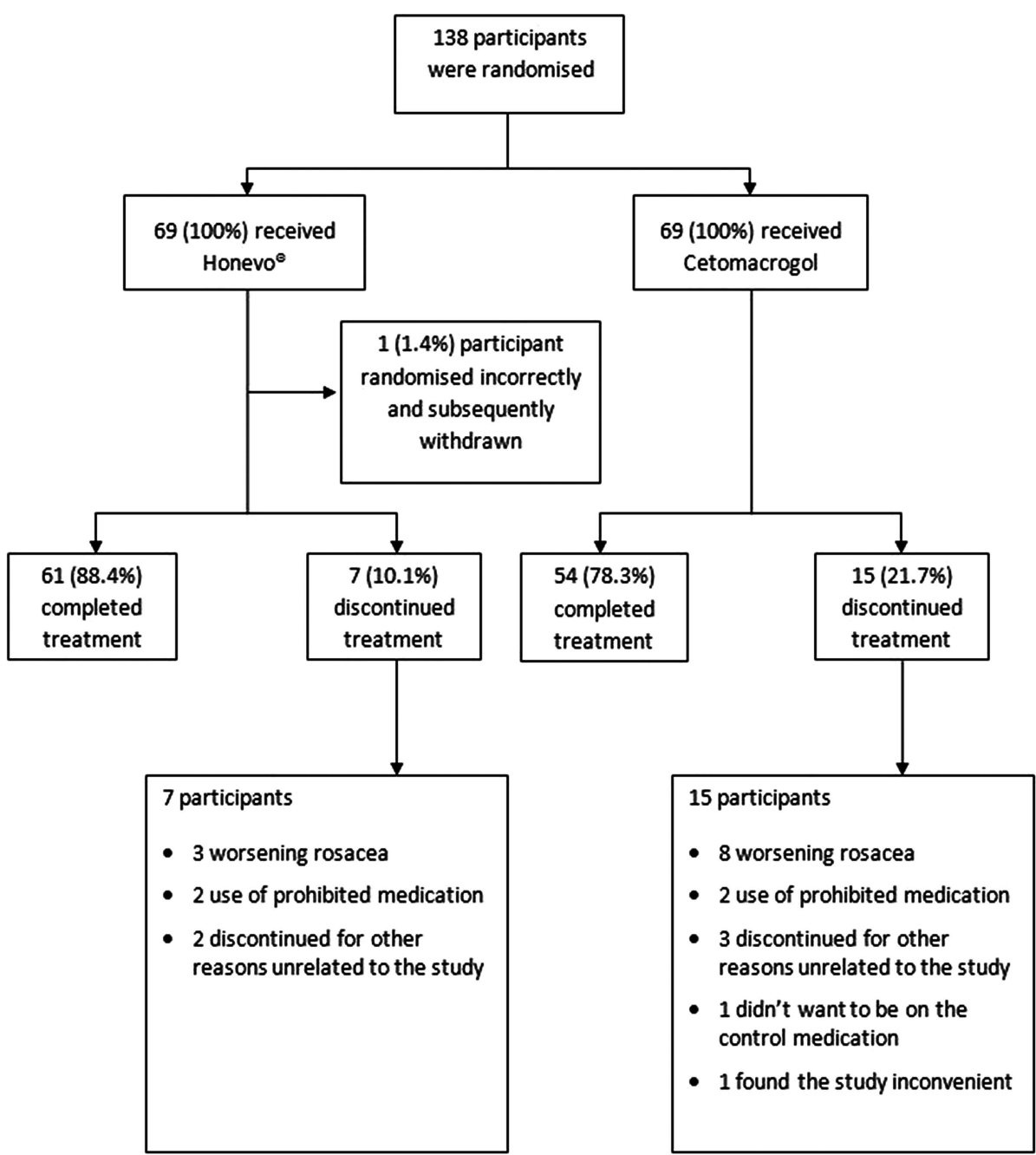

Table 1 Baseline characteristics of participants

\begin{tabular}{lccc}
\hline & Mean (SD) & Median (IQR) & Minimum-maximum \\
\hline $\begin{array}{l}\text { Age at enrolment } \\
\text { Honevo N=68 }\end{array}$ & $57.7(13.7)$ & $58.2(46.7-68.3)$ & \\
Control N=69 & $58.9(15.9)$ & $60.1(49.0-68.6)$ & $18.4-86.5$ \\
All N=137 & $58.3(14.8)$ & $58.9(48.1-68.6)$ & $18.2-90.1$ \\
Age at diagnosis & & & \\
Honevo N=64 & $42.2(15.4)$ & $40(30-51.5)$ & $19-80$ \\
Control N=67 & $43.6(15.4)$ & $43(35-55)$ & $10-79$ \\
All N=131 & $42.9(15.4)$ & $41(32-54)$ & $10-80$ \\
\hline
\end{tabular}

\begin{tabular}{llc}
\hline & Honevo N (\%) & $\begin{array}{c}\text { Control N (\%) } \\
\mathbf{N}=69\end{array}$ \\
\hline Female & $\mathbf{N = 6 8}$ & $36(52.2)$ \\
History of oral antibiotics & $32(47.1)$ & $13(18.8)$ \\
History of topical therapy & $13(19.1)$ & $20(29.0)$ \\
History of topical steroid & $24(35.3)$ & $6(8.7)$ \\
European & $6(8.8)$ & $68(98.6)$ \\
Maori & $64(94.1)$ & $0(0)$ \\
Asian & $4(5.9)$ & $1(1.5)$ \\
\hline
\end{tabular}


Table 2 Contingency table of blinded Investigator Global Assessment of Rosacea Severity Score (IGA-RSS) week 8 change from baseline by randomisation in the participants who completed the study

\section{Change from}

\begin{tabular}{llc} 
baseline & Honevo N/61 (\%) & Control N/54 (\%) \\
\hline-3 & $11(18.0)$ & $1(1.9)$ \\
-2 & $13(21.3)$ & $11(20.4)$ \\
-1 & $20(32.8)$ & $17(31.5)$ \\
0 & $14(23.0)$ & $15(27.8)$ \\
1 & $3(4.9)$ & $8(14.8)$ \\
2 & $0(0)$ & $2(3.7)$
\end{tabular}

IGA-RSS: blinded Investigator Global Assessment of Rosacea Severity Score based on a seven-point scale (0 'clear' to 6 'severe'.

participant could not be blinded due to the appearance and smell of Honevo. It was for this reason we chose to use the seven-point IGA-RSS representing a global assessment of severity of rosacea that was undertaken by an investigator who was blinded to treatment.

The primary outcome variable identified the proportion in each treatment arm who had a two-point reduction or more in IGA-RSS, representing a clinically meaningful improvement in severity of rosacea, for example, a change from 'severe' to 'moderate', or from 'moderate' to 'mild'. Thirty-four per cent of those who were randomised to Honevo had an IGA-RSS improvement of two or more at 8 weeks compared to $17 \%$ of the placebo group, and $13 \%$ achieved full resolution of rosacea compared to $3 \%$ in the placebo group. Although these point estimates are consistent with effectiveness, the CIs are wide which could be consistent with a small effect or quite large effect. This is especially the case for the post hoc analysis of IGA-RSS for the proportion of participants with complete resolution, which although it favours the active treatment, could be consistent with quite a small effect. The estimate of the location shift for the difference between the treatments was $-1,(95 \% \mathrm{CI}-1$ to 0$)$ at both weeks 2 and 8 and the estimated change in IGA-RSS at week 8 adjusted for baseline was $-0.6(95 \% \mathrm{CI}-1.1$ to -0.2$)$. These findings reflect the variability in response to Honevo, perhaps dependent on subtypes of rosacea which were not assessed in this study.

In addition, we assessed patient-reported outcomes, based on participant's assessment of current severity of symptoms (VAS-S), participant's perceived change in severity (VAS-CS), and the DLQI questionnaire, to provide a comprehensive assessment of efficacy. The VAS-CS at the 2-week and 8-week clinic visits was significantly better with Honevo. However, there was no difference with the 2-week and 8-week DLQI assessments or the weekly diary VAS-S measures. Thus, the patient assessments were not completely consistent with the other assessments of efficacy. This could mean that the variability in these led to insufficient statistical power to detect a difference, or in the context of rosacea, that an ideal measurement related to efficacy that is sensitive to change needs further development. The rosacea quality-of-life instrument has been recently reviewed in addition to the DLQI and a generic health-related quality-of-life instrument, the SF-36, in rosacea, and may be a suitable instrument for future research. ${ }^{21}$ In the absence of a validated VAS for rosacea severity, it is difficult to comment on the clinical relevance of a reduction of 11 points from baseline in the VAS-S or the 12.3 improvement in VAS-CS in the Honevo group compared to the control group after 8 weeks. As these outcomes were participant assessed and participants could not be blinded to the interventions, there is a risk of detection
Figure 2 The difference from baseline in IGA-RSS at week 8 for control and Honevo. Horizontal lines are the $25 \%$, median and $75 \%$ quartiles, the symbol is the mean and the whiskers go from maximum to minimum.

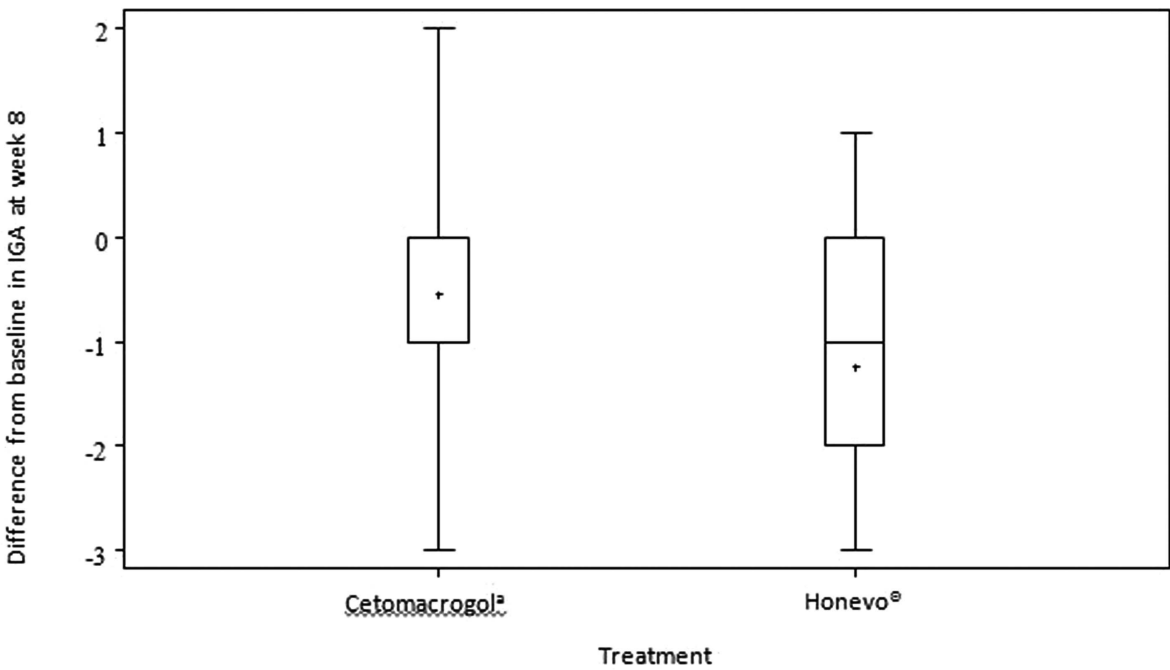

IGA-RSS: Blinded Jnyestigator Global Assessment of Rosacea Severity Score a The median and $25^{\text {th }}$ percentile for Cetomacrogol were the same $(-1)$ 
Table 3 Clinic-based secondary outcome variables (all Honevo minus control)

\begin{tabular}{|c|c|c|c|c|}
\hline \multirow[b]{2}{*}{ Comparison } & \multicolumn{2}{|c|}{$\mathrm{N}$ with data } & \multirow[b]{2}{*}{ Hodges-Lehman estimate $(95 \% \mathrm{CI})$} & \multirow[b]{2}{*}{ p Value } \\
\hline & Honevo & Control & & \\
\hline IGA-RSS week 2 & 66 & 66 & $0(-1$ to 0$)$ & 0.26 \\
\hline IGA-RSS week 8 & 61 & 54 & $0(-1$ to 0$)$ & 0.06 \\
\hline IGA-RSS week 2 minus baseline & 66 & 66 & $-1(-1$ to 0$)$ & 0.03 \\
\hline \multirow[t]{2}{*}{ IGA-RSS week 8 minus baseline } & 61 & 54 & $-1(-1$ to 0$)$ & 0.005 \\
\hline & & & Estimate $(95 \% \mathrm{Cl})$ & p Value \\
\hline \multirow[t]{2}{*}{ IGA-RSS week 8 adjusted for baseline* } & 61 & 54 & $-0.6(-1.1$ to -0.2$)$ & 0.003 \\
\hline & & & Mean difference $(95 \% \mathrm{Cl})$ & p Value \\
\hline VAS-CS week 2 & 66 & 66 & $9.1(3.5$ to 14.7$)$ & 0.002 \\
\hline VAS-CS week 8 & 61 & 54 & $12.3(5.7$ to 18.9$)$ & $<0.001$ \\
\hline VAS-S week 2 & 66 & 66 & $-3.6(-9.9$ to 2.7$)$ & 0.26 \\
\hline VAS-S week 2 minus baseline & 66 & 66 & $-2.9(-9.2$ to 3.5$)$ & 0.38 \\
\hline VAS-S week 8 & 61 & 54 & $-4.5(-11.6$ to 2.6$)$ & 0.21 \\
\hline VAS-S week 8 minus baseline & 61 & 54 & $-11.0(-18.0$ to -3.9$)$ & 0.003 \\
\hline \multicolumn{5}{|c|}{$\begin{array}{l}\text { IGA-RSS: blinded Investigator Global Assessment of Rosacea Severity Score, based on a seven-point scale (0 'clear' to } 6 \text { 'severe'). } \\
\text { VAS-CS: Participant-rated assessment of change in severity of rosacea based on a } 100 \mathrm{~mm} \text { VAS scale ( } 0 \mathrm{~mm} \text { 'much worse' to } 100 \mathrm{~mm} \text { 'much } \\
\text { improved'). } \\
\text { VAS-S: Participant-rated assessment of severity of rosacea based on a } 100 \mathrm{~mm} \text { VAS scale ( } 0 \mathrm{~mm} \text { 'mildest possible' symptoms and } 100 \mathrm{~mm} \\
\text { 'worst possible' symptoms). } \\
\text { Analysis of variance (ANOVA) with baseline reading as a covariate, normality assumptions not well met. } \\
\text { *ANOVA with baseline reading as a co-variate, normality assumptions not well met. }\end{array}$} \\
\hline
\end{tabular}

bias in this methodology. The DLQI is a questionnaire non-specific to rosacea and may not have been sensitive enough to capture changes associated with this condition alone. The drop-out rate for this study was greater than anticipated, which will need to be factored into future research in similar clinical studies. The greater number of withdrawals due to worsening rosacea in the control group ( $12 \%$ vs $4 \%$ ), and overall withdrawals is likely to have led to an underestimation of the efficacy of Honevo, as these participants did not undergo assessment of the secondary outcome variables following withdrawal.

Cetomacrogol cream was chosen as a comparator as it is a non-ionic moisturising cream, often used as a vehicle for delivery of topical medications. ${ }^{22}{ }^{23}$ The treatment was administered for 8 weeks to allow both the speed of onset and the duration of effect to be assessed. This identified that honey had efficacy within 2 weeks of treatment, with further efficacy obtained during the 8-week treatment period. Adverse event data did not allow us to reliably ascertain whether the rosacea-specific adverse events such as burning, itching, peeling, stinging, dry skin and pain were associated only with the application of the investigational medicine or whether they were ongoing rosacea symptoms. However, it was reassuring that the number of participants with rosacea-specific adverse events was similar between Honevo and the paraffin-based emollient control cream.

Notwithstanding the limitations of comparing different primary outcome variables utilised in other studies of rosacea, it is interesting to note the findings from placebo-controlled studies of topical metronidazole or azelaic acid, two of the more commonly used treatments for rosacea. ${ }^{4-7}$ In studies of topical metronidazole, the relative risk of improvement with the physician's global evaluation of improvement was 1.95 (95\% CI 1.5 to 2.6), and in studies of azelaic cream the relative risk of participant-assessed improvement was 1.52 (95\% CI 1.3 to 1.8$).{ }^{64}$ In our study, the relative risk of IGA-RSS improvement with Honevo was 2.0 (95\%CI 1.1 to 3.7), and in the post hoc analysis the relative risk of resolution was 4.6 (95\% CI 1.0 to 20.4). It is difficult to indirectly compare the results of these studies of two other active treatments against placebo with the study reported here, as the characteristics of the research participants may have been quite different and the outcome variable of global improvement may not correspond to that used in this study. Despite this, we have found sufficient evidence of efficacy, albeit with wide CIs, that a reasonable next stage is to conduct randomised controlled trials comparing Honevo with topical metronidazole or azelaic cream.

The mechanism of action was not assessed in this study, however, there are a number of potential mechanisms relevant to the efficacy demonstrated with kanuka honey in this study. First, kanuka honey has a number of different anti-inflammatory effects, including inhibition of neutrophil superoxide production, ${ }^{13}$ reduction in inflammatory leucocyte infiltration and arachidonicinduced oedema, ${ }^{13}$ and stimulation of macrophage release of tumour necrosis factor $\alpha,{ }^{15}$ a cytokine with a crucial role in wound healing. ${ }^{25} 26$ These properties may be relevant as rosacea is a chronic inflammatory disorder, characterised by inflammatory cell infiltration, vascular dilation and tissue oedema. ${ }^{1}{ }^{3}{ }^{6}$ In addition, kanuka honey has high antibacterial activities against a 
wide range of bacteria, including Bacillus subtilis, Propionibacterium acne and Staphylococcus aureus, ${ }^{11} 12$ 14 properties which may be beneficial in view of the proposed role of $B$. oleronius in the inflammatory response in rosacea. The effect of honey on $B$. oleronius and the Demodex folliculum mite requires further investigation.

In conclusion, this randomised controlled trial has demonstrated the clinical efficacy and tolerability of $90 \%$ medical-grade kanuka honey and $10 \%$ glycerine (Honevo) in the treatment of rosacea. Honevo can be recommended for the treatment of rosacea; however, further randomised controlled trials comparing Honevo with topical metronidazole and azelaic acid are now required to determine its relative efficacy and side effect profile compared to these agents.

Guarantor: Dr I Braithwaite had access to all the data on the study and takes responsibility for the integrity of the data and accuracy of the data analysis.

Contributors JF, JK, MH, MW and RB contributed to study concept and design. IB, AH, JR, AC, CH, DS, CT and BM contributed to acquisition of the data. RB, IB and MW drafted the manuscript. All the authors contributed to critical revision of the manuscript for important intellectual content and also to administrative, technical and material support. MW contributed to statistical analysis. IB is the guarantor.

Funding This study was funded by HoneyLab. HoneyLab provided the Honevo (medical-grade kanuka honey and 10\% glycerine) for the study.

Competing interests None declared.

Patient consent Obtained.

Ethics approval The New Zealand Health and Disability Ethics Committee.

Provenance and peer review Not commissioned; externally peer reviewed.

Data sharing statement Patient-level data available from the corresponding author. Consent was not obtained from participants for data sharing, but the presented data are anonymised and the risk of identification is low. Data may be obtained by contacting the corresponding author.

Open Access This is an Open Access article distributed in accordance with the Creative Commons Attribution Non Commercial (CC BY-NC 4.0) license, which permits others to distribute, remix, adapt, build upon this work noncommercially, and license their derivative works on different terms, provided the original work is properly cited and the use is non-commercial. See: http:// creativecommons.org/licenses/by-nc/4.0/

\section{REFERENCES}

1. Buechner SA. Rosacea: an update. Dermatology 2005;210:100-8.

2. Wilkin J, Dahl M, Detmar M, et al. Standard grading system for rosacea: report of the National Rosacea Society Expert Committee on the classification and staging of rosacea. J Am Acad Dermatol 2004;50:907-12.

3. Berg M, Liden S. An epidemiological study of rosacea. Acta Derm Venereol 1989;69:419-23.

4. Powell FC. Clinical practice. Rosacea. N Engl J Med 2005;352:793-803.
5. Elewski BE, Fleischer AB Jr, Pariser DM. A comparison of $15 \%$ azelaic acid gel and $0.75 \%$ metronidazole gel in the topical treatment of papulopustular rosacea: results of a randomized trial. Arch Dermatol 2003;139:1444-50.

6. van Zuuren EJ, Kramer S, Carter B, et al. Interventions for rosacea. Cochrane Database Syst Rev 2011;(3):CD003262.

7. Elewski BE. Rosacea trial comparing twice-daily azelaic acid gel $15 \%$ with once-daily metronidazole gel $1 \%$. Cutis 2007;79:57-8; author reply 8 .

8. World Health O. Antimicrobial resistance: global report on surveillance 2014. 2014; Report No. ISBN: 9789241564748 Contract No. Document Number.

9. Costelloe C, Metcalfe C, Lovering A, et al. Effect of antibiotic prescribing in primary care on antimicrobial resistance in individual patients: systematic review and meta-analysis. $B M J$ 2010;340:c2096.

10. Department of Health UKSMAC. The path of least resistance. http:// antibiotic-action.com/wp-content/uploads/2011/07/Standing-MedicalAdvisory-Committee-The-path-of-least-resistance-1998.pdf [accessed January 2015] 1998.

11. Wu Q. Antimicrobial effect of Manuka honey and Kanuka honey alone and in combination with the bioactives against the growth of Propionibacterium acnes ATCC 6919: a thesis submitted in partial fulfilment of the requirements for the degree Master of Food Technology. Albany, New Zealand, Massey University, 2011.

12. Lu J, Carter DA, Turnbull L, et al. The effect of New Zealand kanuka, manuka and clover honeys on bacterial growth dynamics and cellular morphology varies according to the species. PLOS ONE 2013;8:e55898.

13. Leong AG, Herst PM, Harper JL. Indigenous New Zealand honeys exhibit multiple anti-inflammatory activities. Innate Immun 2012;18:459-66.

14. Allen KL, Molan PC, Reid GM. A survey of the antibacterial activity of some New Zealand honeys. J Pharm Pharmacol 1991;43:817-22.

15. Gannabathula S, Skinner MA, Rosendale D, et al. Arabinogalactan proteins contribute to the immunostimulatory properties of New Zealand honeys. Immunopharmacol Immunotoxicol 2012;34:598-607.

16. Lacey N, Delaney S, Kavanagh $\mathrm{K}$, et al. Mite-related bacterial antigens stimulate inflammatory cells in rosacea. Br J Dermatol 2007;157:474-81.

17. Bonnar E, Eustace P, Powell FC. The Demodex mite population in rosacea. J Am Acad Dermatol 1993;28:443-8.

18. Yamasaki K, Di Nardo A, Bardan A, et al. Increased serine protease activity and cathelicidin promotes skin inflammation in rosacea. Nat Med 2007;13:975-80.

19. Fingleton JS, Cave D, Brinded N, et al. Topical kanuka honey for the treatment of rosacea. Focus Altern Complement Ther 2013;18:221-2.

20. Lewis V, Finlay AY. 10 years experience of the Dermatology Life Quality Index (DLQI). J Investig Dermatol Symp Proc 2004:9:169-80.

21. Van der Linden MM, van Rappard DC, Daams JG, et al. Health-related quality of life in patients with cutaneous rosacea a systematic review. Acta Derm Venereol 2015;95:395-400.

22. (Datapharm) ew. Cetomacrogol Cream BP 1988 Formula A. https:// wwwmedicinesorguk/emc/medicine/25140 (accessed 24 Dec 2014).

23. Kingdom NNHSU. Cetomacrogol Cream Formula A. http://wwwnhsuk/ medicine-guides/pages/MedicineOverviewaspx?medicine= Cetomacrogol\%20Cream\%20Formula\%20A (accessed 24 Dec 2014).

24. van Zuuren EJ, Kramer SF, Carter BR, et al. Effective and evidence-based management strategies for rosacea: summary of a Cochrane systematic review. Br J Dermatol 2011;165:760-81.

25. Fu X, Tian H, Hsu S, et al. In vivo effects of tumor necrosis factor-alpha on incised wound and gunshot wound healing. J Trauma 1996;40(3 Suppl):S140-3.

26. Mooney DP, O'Reilly M, Gamelli RL. Tumor necrosis factor and wound healing. Ann Surg 1990;211:124-9. 